\title{
Optical conductivity in $\mathrm{A}_{3} \mathrm{C}_{60}(\mathrm{~A}=\mathrm{K}, \mathrm{Rb})$
}

\author{
J. van den Brink ${ }^{1,2}$, O. Gunnarsson ${ }^{1}$ and V. Eyert ${ }^{1,3}$ \\ ${ }^{1}$ Max-Planck-Institut für Festkörperforschung, D-70506 Stuttgart, Germany \\ ${ }^{2}$ Laboratory of Solid State and Applied Physics, Materials Science Centre, University of Groningen, Nijenborgh 4, 9747 AG \\ Groningen, The Netherlands \\ ${ }^{3}$ Hahn-Meitner-Institut, Glienicker Straße 100, D-14109 Berlin, Germany
}

(August 13, 2018)

\begin{abstract}
We study the optical conductivity in $\mathrm{A}_{3} \mathrm{C}_{60}(\mathrm{~A}=\mathrm{K}, \mathrm{Rb})$. The effects of the electron-phonon interaction is included to lowest order in the coupling strength $\lambda$. It is shown that this leads to a narrowing of the Drude peak by a factor $1+\lambda$ and a transfer of weight to a mid-infrared peak at somewhat larger energies than the phonon energy. Although this goes in the right direction, it is not sufficient to describe experiment.
\end{abstract}

\section{INTRODUCTION}

The optical conductivity in $\mathrm{A}_{3} \mathrm{C}_{60}(\mathrm{~A}=\mathrm{K}, \mathrm{Rb})$ has an unusual and interesting behaviour. 0 The weight of the Drude peak is reduced by one order of magnitude relative to the weight for free electrons with the appropriate band mass. Much of the missing weight appears instead in a "mid-infrared" structure at about $0.06 \mathrm{eV}$. This suggests very strong interaction effects, e.g., electron-phonon or Coulomb interaction. The understanding of the optical absorption could therefore contribute much to the understanding also of other properties of $\mathrm{A}_{3} \mathrm{C}_{60}$.

$\mathrm{A}_{3} \mathrm{C}_{60}$ has orientational disorder, with the $\mathrm{C}_{60}$ molecules taking, more or less randomly, one out of two preferential orientations. 6 This orientational disorder leads to a substantial modification of the optical conductivity in one-particle calculations. For an ordered system, the Drude peak collapses to a $\delta$-function, while the disorder leads to a broad Drude peak 0 The calculated optical conductivity furthermore shows a structure at somewhat larger energies than the experimental midinfrared structure, although the structure is less pronounced and at higher energy than in the experimental spectrum. More serious is, however, that the weight and width of the Drude peak are much larger than the experimental results. Although it is hard to separate the theoretical results in a Drude and a mid-infrared structure, it may be estimated that the theoretical Drude width is more than a factor of ten too large.

The strong reduction of the Drude width suggests very strong renormalization effects, e.g., due to the electronphonon or electron-electron interactions. The fullerenes have phonons with an energy of about $006 \mathrm{eV}$, which show a strong coupling to the electrons $\mathrm{O}$ Since these phonons may transfer weight from the Drude peak to a mid-infrared structure, we here study the effect of phonons.

We limit ourselves to calculating the electron selfenergy tp lowest order. This is sufficient if Migdal's theorem $\mathrm{l}$ is valid. It is, however, questionable if this is true for the fullerenes, and we should keep in mind that higher order effects may be important. To obtain the optical conductivity we calculate the current-current response function. We can neglect vertex corrections, 10 since the electron self-energy is q-independent in our approach. The current-current response function is then reduced to a product of two electron Green's function. We find that the electron-phonon coupling leads to a narrowing of the Drude peak by about a factor of $1 /(1+\lambda)$, where $\lambda$ is the is the electron-phonon coupling constant. Although this goes in the right direction, it is by far not sufficient to explain the experimental data.

In Sec. II we present the formalism and the model. In Sec. III we show the results and in Sec. IV multiplet effects are briefly discussed. The results and other possible explanations of the narrow Drude peak are discussed in Sec. V.

\section{FORMALISM AND MODEL}

The optical conductivity is given by 11

$$
\operatorname{Re} \sigma_{\alpha \beta}(\omega)=\operatorname{Re}_{\mathbf{q} \rightarrow 0} \lim _{\frac{i}{\omega}} \pi_{\alpha \beta}(\mathbf{q}, \omega)
$$

where

$$
\pi_{\alpha \beta}(\mathbf{q}, \omega)=-i \int_{0}^{\infty} d t e^{i \omega t}\left\langle 0\left|\left[j_{\alpha}^{\dagger}(\mathbf{q}, t), j_{\beta}(\mathbf{q}, 0)\right]\right| 0\right\rangle .
$$

Here $j$ is the current operator and $|0\rangle$ is the ground-state. Below, we use a formalism where the electron self-energy is $\mathbf{q}$-independent. It can then be shown ${ }^{10}$ that the vertex corrections in the current-current response function vanish for $\mathbf{q} \rightarrow 0$, due to the odd parity of the current operator. We can then write the optical conductivity as a product of two Green's functions, only keeping a simple bubble of dressed Green's functions in the diagrammatic expansion of $\sigma$. If we express the current operator as

$$
j_{\alpha}=\sum_{\sigma} \sum_{n n^{\prime}} v_{n n^{\prime}}^{\alpha} c_{n \sigma}^{\dagger} c_{n^{\prime} \sigma}
$$


the optical conductivity is given by

$$
\begin{aligned}
\operatorname{Re} \sigma_{\alpha \beta} & =\frac{2}{\omega} \operatorname{Re} \sum_{n n^{\prime}} \sum_{m m^{\prime}} v_{n n^{\prime}}^{\alpha *} v_{m m^{\prime}}^{\beta} \\
& \times \int_{-\infty}^{\infty} \frac{d \omega^{\prime}}{2 \pi} G_{n m^{\prime}}\left(\omega+\omega^{\prime}\right) G_{m n^{\prime}}\left(\omega^{\prime}\right)
\end{aligned}
$$

where $G$ is the electron Green's function. This can be rewritten as 12

$$
\begin{aligned}
& \operatorname{Re} \sigma_{\alpha \beta}=\frac{2 \pi}{\omega} \sum_{n n^{\prime}} \sum_{m m^{\prime}} v_{n n^{\prime}}^{\alpha *} v_{m m^{\prime}}^{\beta} \\
& \times \int_{-\infty}^{\infty} d \omega^{\prime} A_{n m^{\prime}}\left(\omega+\omega^{\prime}\right) A_{m n^{\prime}}\left(\omega^{\prime}\right)\left[f\left(\omega^{\prime}\right)-f\left(\omega^{\prime}+\omega\right)\right]
\end{aligned}
$$

where $A_{n m}(\omega)=\operatorname{Im} G_{n m}\left(\omega-i 0^{+}\right) / \pi$ and $f(\omega)$ is the Fermi function.

We consider the three $t_{1 u}$ orbitals of $\mathrm{C}_{60}$ which are connected by hopping matrix elements $t$

$$
H^{\mathrm{el}}=\sum_{i \sigma} \sum_{m=1}^{3} \varepsilon_{t_{1 u}} n_{i m \sigma}+\sum_{<i j>\sigma m m^{\prime}} t_{i j m m^{\prime}} \psi_{i m \sigma}^{\dagger} \psi_{j m^{\prime} \sigma}
$$

The orientational diserden 6 has been built into the matrix elements $t_{i j m m^{\prime}} 1315$ Deshpande et al. have used a similar model for calculating the phonon self-energy. 16 We want to describe the coupling to the intramolecular five-fold degenerate $H_{g}$ Jahn-Teller modes. Due to the intramolecular character, the coupling has a local form. To describe the electron-phonon interaction, we use the Hamiltonian

$$
\begin{aligned}
H^{\mathrm{el}-\mathrm{ph}} & =\omega_{p h} \sum_{m=1}^{5}\left(b_{m}^{\dagger} b_{m}+\frac{1}{2}\right) \\
& +\frac{g}{2} \sum_{m=1}^{5} \sum_{\sigma} \sum_{i=1}^{3} \sum_{j=1}^{3} V_{i j}^{(m)} \psi_{i \sigma}^{\dagger} \psi_{j \sigma}\left(b_{m}+b_{m}^{\dagger}\right),
\end{aligned}
$$

where $\omega_{p h}$ is the a phonon frequency, $b_{m}$ annihilates a phonon with quantum number $m, V_{i j}^{(m)}$ are dimensionless coupling constants 17 given by symmetry and $g$ is an overall coupling strength. The electron-phonon coupling constant $\lambda$ is then given by

$$
\lambda=\frac{5}{3} N(0) \frac{g^{2}}{\omega_{p h}}
$$

where $N(0)$ is the density of states per spin at the Fermi energy.

We now construct a consistent current operator, essentially following Ref. 19. We write the density $\rho(i)$ at a site $i$ as

$$
\rho(i)=\sum_{m \sigma} \psi_{i m \sigma}^{\dagger} \psi_{i m \sigma}
$$

Here we only consider the number of electrons on a given site, and neglect the possible polarization of the charge on this $\mathrm{C}_{60}$ molecule. Due to this assumption we obtain no terms in the current operator describing on-site transitions. Since the transitions between $t_{1 u}$ orbitals on the same site are forbidden, Eq. (11) is sufficient for our purposes. Imposing charge and current conservation

$$
\mathbf{q} \cdot \mathbf{j}(\mathbf{q})=-e[H, \rho(\mathbf{q})]
$$

we obtain

$$
\mathbf{q} \cdot \mathbf{j}(\mathbf{q})=-\frac{i e}{\sqrt{N}} \sum_{i j m m^{\prime}} t_{i j m m^{\prime}} \mathbf{q} \cdot\left(\mathbf{R}_{i}-\mathbf{R}_{j}\right)
$$

in the limit $\mathbf{q} \rightarrow 0$. Here $\mathbf{R}_{i}$ is the position of molecule $i$. We then obtain the current matrix elements

$$
v_{i m, j m^{\prime}}^{\alpha}=-i e t_{i j m m^{\prime}}\left(R_{i}^{\alpha}-R_{j}^{\alpha}\right) .
$$

The electron self-energy is calculated to lowest order in the electron-phonon interaction.

$$
\Sigma_{n n^{\prime}}^{\mathrm{El}-\mathrm{phon}}(\omega)=i \sum_{m \mu} \int \frac{d \omega^{\prime}}{2 \pi} \lambda_{n m}^{\mu} G_{m m}^{(0)}\left(\omega-\omega^{\prime}\right) D_{\mu \mu}^{(0)}\left(\omega^{\prime}\right) \lambda_{m n^{\prime}}^{\mu}
$$

where $G_{m m}^{(0)}$ and $D_{\mu \mu}^{(0)}$ are the zero order electron and phonon Green's functions, respectively. The electronphonon coupling is described by $\lambda_{n m}^{\mu}$, which is expressed in terms of the coupling constants $V_{i j}^{(m)}$ and the oneparticle solutions. The interacting electron Green's function is then obtained from the Dyson's equation

$$
G(\omega)=G^{(0)}(\omega)+G^{(0)}(\omega) \Sigma(\omega) G(\omega),
$$

where a matrix notation has been used.

We next discuss qualitatively how the optical conductivity may change due to the electron-phonon interaction. If the band width is much larger than a typical phonon frequency, Migdal's theorem 9 is valid. For states with an energy smaller than the phonon energy, the quasiparticle energy is then reduced by a factor 20

$$
Z=\frac{1}{1+\lambda}
$$

where $\lambda$ is the electron-phonon coupling. Furthermore the quasiparticle weight is reduced by the same factor. 20 For $\mathrm{A}_{3} \mathrm{C}_{60}$ it is very questionable if Migdal's theorem is valid, and interesting effects happen due to the fact that the band width is not much larger than the phonon frequencies.21 Nevertheless, we can expect to obtain some insight into the effect of the electron-phonon interaction by making the above assumptions, i.e., assuming that the electrons can be treated as noninteracting but with 
weights and energies which are reduced by a factor $Z$. For $\omega>0$ we then have

$$
\sigma_{\alpha \alpha}(\omega) \sim \lim _{\mathbf{q} \rightarrow 0} \frac{1}{\omega} \operatorname{Im} \sum_{n}^{\text {unocc occ }} \sum_{m}^{\text {occ }} \frac{\left|\left\langle n\left|j_{\alpha}(\mathbf{q})\right| m\right\rangle\right|^{2}}{\omega-\varepsilon_{n}+\varepsilon_{m}-i 0^{+}}
$$

We replace $\varepsilon_{n}$ by $Z \varepsilon_{n}^{(0)}$ and $\left\langle n\left|j_{\alpha}(\mathbf{q})\right| m\right\rangle \quad$ by $Z\left\langle n\left|j_{\alpha}(\mathbf{q})\right| m\right\rangle^{(0)}$, where the suffix 0 refers to the noninteracting system. This leads to

$$
\sigma_{\alpha \alpha}(\omega)=\sigma_{\alpha \alpha}^{(0)}\left(\frac{\omega}{Z}\right)
$$

where $\sigma^{(0)}$ is the optical conductivity without the electron-phonon interaction. For zero frequency $\sigma$ is unchanged, as it should, since the resistivity $\sigma(0)$ is not influenced by the electron-phonon interaction at zero temperature, considered here. We can see, however, that the energy scale is reduced by a factor of $Z$, and that the weight of the Drude peak is reduced correspondingly. For larger frequencies these considerations are of course too simple, since we then have to consider the whole Green's function including phonon satellites and not just the quasiparticle.

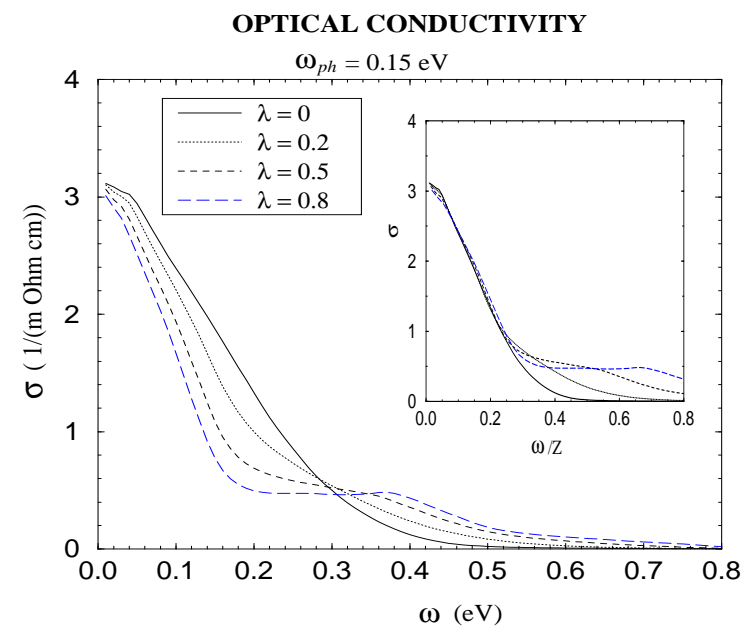

FIG. 1. Optical conductivity $\sigma(\omega)$ for the phonon frequency $\omega_{p h}=0.15 \mathrm{eV}$ and for different electron-phonon coupling constants $\lambda$. The figure illustrates how the Drude peak becomes narrower and how weight is transferred to a mid-infrared peak as $\lambda$ is increased. The inset shows $\sigma$ as a function of $\omega / Z$, where $Z=1+\lambda$. This illustrates how the width of the Drude peak is reduced by a factor of $1+\lambda$ due to the electron-phonon interaction.

\section{RESULTS}

In Fig. 1 1 we show the optical conductivity for a phonon frequency $\omega_{p h}=0.15 \mathrm{eV}$. Without electron-phonon coupling $(\lambda=0)$ the spectrum shows a broad Drude peak. As $\lambda$ is increased, the Drude peak becomes narrower and weight is transferred to a structure in the energy range 0.2-0.4 eV. In the inset in Fig. 1 the same results are shown as a function of $\omega / Z$. The curves now essentially fall on top of each other for small $\omega$. This illustrates the result (19) that the width of the Drude peak is reduced by a factor $Z$. Fig. 2 shows the results for a lower phonon frequency $\omega_{p h}=0.05 \mathrm{eV}$. The spectrum is similar as in Fig. 1, but the mid-infrared structure has moved to lower frequencies.

\section{OPTICAL CONDUCTIVITY}

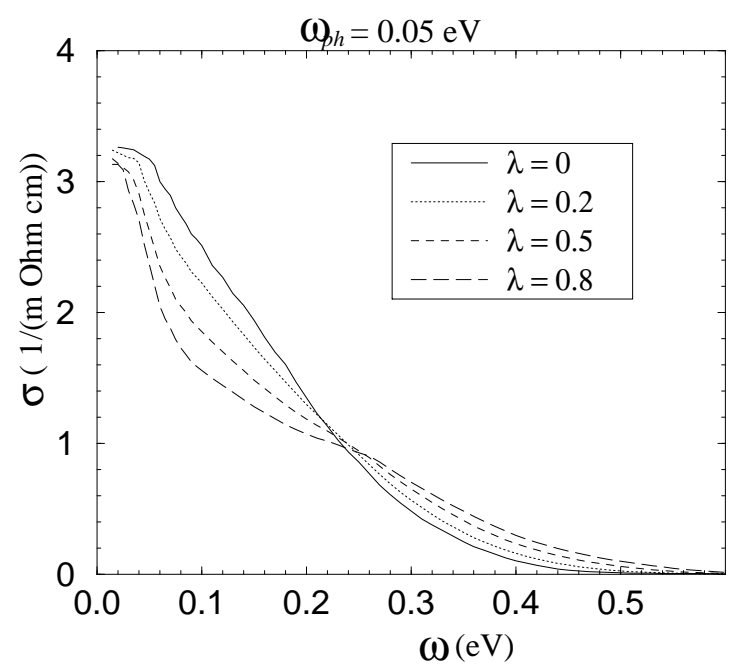

FIG. 2. The same as in Fig. 1 but as a function of $\omega / Z$, where $Z=1 /(1+\lambda)$. The similarity of the curves for small values of $\omega / Z$ but different values of $\lambda$ illustrates how the Drude peak width is reduced by a factor $Z$.

From photoemission for a free $\mathrm{C}_{60}^{-}$molecule 22 and from neutron scattering 23 it has been estimated that the strongest coupling is to the second lowest $\mathrm{H}_{g}$ mode at about $0.054 \mathrm{eV}$. From Raman scattering the strongest coupling was found for the lowest mode at about $\omega_{p h}=$ $0.033 \mathrm{eV} 24$ The value $\omega_{p h}=0.05$ used in Fig. 2 should therefore be more realistic then the one in Fig. 1, and one might even argue for a still smaller value of $\omega_{p h}$. This would then tend to give an energy of the midinfrared structure of the right order of magnitude, although it is still larger than the experimentally observed value $0.06 \mathrm{eV}$. The electron-phonon coupling is of the order $\lambda \sim 0.5-1.0$. The width of the Drude peak is then reduced by a factor of 1.5-2. This reduction goes in the right direction, but it is much too small to explain experiment.

\section{MULTIPLET EFFECTS}

An alternative mechanism for transferring weight from the Drude peak to the mid-infrared peak is provided by multiplet effects. Within the $t_{1 u}$ system, these are described by the exchange integral $K$ between two $t_{1 u}$ orbitals and the difference $\delta U \equiv U_{x x}-U_{x y}$ between the 
direct Coulomb integral for equal and unequal orbitals. Here we use $\delta U=2 K$. The $\mathrm{C}_{60}^{3-}$ molecule has a groundstate with spin $3 / 2$ and states with the spin $1 / 2$ at $3 K$ and $5 K$ above the ground-state. The value of $K$ has been estimated to be $0.05 \mathrm{eV}, 25$ and $0.024 \mathrm{eV} .26$ The unscreened value has been found to be $K=0.12 \mathrm{eV}$ and within RPA screening $K=0.030 \mathrm{eV}$.27 The experience from atomic multiplets is that these are only weakly reduced $(\sim 20 \%)$ relatively to what is predicted by the unscreened Coulomb integrals, both for free atoms and for solids.28 We also find that to describe the multiplets in the $h_{u}-t_{1 u}$ exciton, unscreened integrals give a splitting of the right order of magnitude. Due to the lack of extensive experience for the large $\mathrm{C}_{60}$ molecule, we nevertheless consider the whole range of estimates for the multiplet integrals below. If the lower values of these estimates are used, the multiplet splitting is of the same order of magnitude as the energy of the mid-infrared structure, and it is then interesting to study to what extent these effects can explain this structure.

We have added a multiplet interaction to the Hamiltonian in Eq. (7)

$$
\begin{aligned}
H_{U} & =\frac{2}{3} \delta U \sum_{i m} n_{i m \uparrow} n_{i m \downarrow}-\frac{1}{3} \delta U \sum_{i \sigma \sigma^{\prime}} \sum_{m<m^{\prime}} n_{i \sigma m} n_{i \sigma^{\prime} m^{\prime}} \\
& +\frac{1}{2} K \sum_{i \sigma \sigma^{\prime}} \sum_{m \neq m^{\prime}} \psi_{i \sigma m}^{\dagger} \psi_{i \sigma^{\prime} m^{\prime}}^{\dagger} \psi_{i \sigma^{\prime} m} \psi_{i \sigma m^{\prime}} \\
& +\frac{1}{2} K \sum_{i \sigma} \sum_{m \neq m^{\prime}} \psi_{i \sigma m}^{\dagger} \psi_{i-\sigma m}^{\dagger} \psi_{i-\sigma m^{\prime}} \psi_{i \sigma m^{\prime}}
\end{aligned}
$$

The simple Coulomb interaction

$$
H_{U}^{0}=U \sum_{i} \sum_{(\sigma m)<\left(\sigma^{\prime} m^{\prime}\right)} n_{i \sigma m} n_{i \sigma^{\prime} m^{\prime}}
$$

should also be added but is not considered here, since in simple treatments it does not give a contribution to the mid-infrared structure.

We have estimated the self-energy to second order in $\delta U$ and $K$ and obtained

$$
\Sigma_{n n \sigma}^{\mathrm{Mult}} \sim \frac{K^{2}}{W}
$$

This has to be compared with the self-energy due to the electron-phonon energy, which is of the order

$$
\Sigma_{n n \sigma}^{\text {El-phon }} \sim \lambda \omega_{p h} .
$$

If we put $K=0.03 \mathrm{eV}, W=0.5 \mathrm{eV}, \lambda=1$ and $\omega_{p h}=0.1$ $\mathrm{eV}$, we find that $\Sigma^{\mathrm{El}-\text { phon }}$ is about a factor of 35 larger than $\Sigma^{\text {Mult }}$. This suggests that although the multiplet effects may transfer weight to the mid-infrared peak, the effect should be very small. If, on the other hand, we use a large value $K=0.15 \mathrm{eV}$ for the multiplet integral, the second order self-energy due to the multiplet integrals becomes comparable to the electron-phonon contribution. In this case, however, the multiplet splitting is much larger than the energy of the mid-infrared peak. It therefore seems likely that the multiplet effects treated in second order theory cannot explain the energy and weight of the mid-infrared peak. We observe, however, the second order perturbation theory used here is not sufficient to describe the atomic limit, and that a better treatment conceivably could change the conclusions somewhat.

\section{DISCUSSION}

We have calculated the optical conductivity, including the effects of the lowest order self-energy diagram due to the electron-phonon interaction. This coupling reduces the width of the Drude peak and transfers weight to the mid-infrared structure at an energy somewhat larger than the phonon frequency. This leads to a mid-infrared structure with an energy of the right order of magnitude, but a bit too large. The reduction of the width of the Drude peak goes in the right direction, but it is much too small. We observe that the self-energy was calculated under the assumption that Migdal's theorem is valid. Since Migdal's theorem is questionable for these systems, higher order corrections could modify these conclusions.

It is interesting that Liechtenstein et al.29 found a rather narrow Drude peak (width $\sim$ a few hundredths of an eV) in a one-particle calculation. As mentioned before, the $\mathrm{C}_{60}$ molecules in $\mathrm{A}_{3} \mathrm{C}_{60}$ have primarily two different orientations. It has been found on theoretical grounds that it is energetically favorable if neighboring $\mathrm{C}_{60}$ molecules have different ("antiferromagnetic") orientations 13.15 The system can then be mapped onto a frustrated Ising model, for which the ground-state has a frustrated antiferromagnetic ordering.15 This ordering leads to the narrowing of the Drude peak mentioned above.29 Experimentally, a tendency to a short-ranged "antiferromagnetic" correlation has been found 30 but under normal experimental conditions the samples are apparently cooled too fast to develop the long-ranged partial order assumed in Ref. 29. It therefore does not seem likely that the partial ordering assumed in Ref. 29 explains the narrow Drude peak in experimental samples used so far.

It is interesting to ask what other effects may contribute to the explanation of the optical conductivity. We have illustrated that multiplet effects are unlikely to explain the experimental results, at least if they are treated to lowest order. These systems have a strong coupling to a charge carrier plasmon at $0,5 \mathrm{eV}$ due to the oscillations of the three $t_{1 u}$ electrons.31.32.21 In analogy with the coupling to the phonons, one may argue that the plasmons have a coupling constant $\lambda_{p l} \sim 2.5 .33$ Taking over the arguments from the electron-phonon coupling one might then expect a substantial narrowing from the coupling to the plasmons. This picture is, however, too simple, and a calculation of the electron self-energy in the so-called GW 
approximation 34 shows only a modest reduction of the band width. 33 Actually, estimates of the specific heat 35,36 do not show an enhancement compared with the result obtained from band structure calculations, apart from the enhancement expected from an electron-phonon interaction with a $\lambda \sim 0.5-1$. If these estimates are correct, they suggest that many-body interactions do not reduce the dispersion in $\mathrm{A}_{3} \mathrm{C}_{60}(\mathrm{~A}=\mathrm{K}, \mathrm{Rb})$. This is also consistent with the susceptibility, 35 which shows a very weak temperature dependence, implying that there is no narrow peak in the density of states. We should then not expect an explanation of the narrow Drude peak in terms of a mechanism which reduces the dispersion beyond the reduction due to the electron-phonon interaction. Instead we should search for a mechanism which influences a two-particle spectrum, like the optical conductivity, without increasing the effective mass.

\section{ACKNOWLEDGEMENTS}

JvdB thanks the Stichting Scheikundig Onderzoek Nederland for financial support.

${ }^{1}$ Y. Iwasa, K. Tanaka, T. Yasuda, T. Koda, and S. Koda, Phys. Rev. Lett. 69, 2284 (1992).

${ }^{2}$ L. Degiorgi, P. Wachter, G. Grüner, S.-M. Huang, J. Wiley, and R.B. Kaner, Phys. Rev. Lett. 69, 2987 (1992).

${ }^{3}$ L. Degiorgi, E.J. Nicol, O. Klein, G. Grüner, P. Wachter, S.-M. Huang, J. Wiley, and R.B. Kaner, Phys. Rev. 49, 7012 (1994).

${ }^{4}$ L. Degiorgi, G. Briceno, M.S. Fuhrer, A. Zettl, and P. Wachter, Nature 369, 541 (1994).

${ }^{5}$ L. Degiorgi, Mod. Phys. Lett. B 9, 445 (1995).

${ }^{6}$ P.W. Stephens, L. Mihaly, P.L. Lee, R.L. Whetten, S.-M. Huang, R. Kaner, F. Deiderich, and K. Holczer, 1991, Nature 351, 632.

${ }^{7}$ M.P. Gelfand and J.P. Lu, Phys. Rev. B 46, 4367 (1992); Phys. Rev. B 47, 4149 (1993). In this paper unrealistically small hopping matrix elements were used. This influences, however, only the energy scale of the spectrum but not its shape. Here we refer to similar calculations with hopping matrix elements giving a $t_{1 u}$ band width of $0.6 \mathrm{eV}$.

${ }^{8}$ O. Gunnarsson, Rev. Mod. Phys. (in press).

${ }^{9}$ A.B. Migdal, Soviet Phys. JETP 1, 996 (1958).

${ }^{10}$ A. Khurana, Phys. Rev. Lett. 64, 1990 (1990); A. Georges, G. Kottliar, W. Krauth, and M.J. Rozenberg, Rev. Mod. Phys. 68, 13 (1996); S. Engelsberg and J.R. Schrieffer, Phys. Rev. 131, 993 (1963).
${ }^{11}$ G.D. Mahan, Many-particle physics, (Plenum, New York, 1983).

${ }^{12}$ H. Schweitzer and G. Czycholl. Phys. Rev. Lett. 67, 3724 (1991).

13 O. Gunnarsson, S. Satpathy, O. Jepsen, and O.K. Andersen, Phys. Rev. Lett. 67, 3002 (1991).

${ }^{14}$ S. Satpathy, V.P. Antropov, O.K. Andersen, O. Jepsen, O. Gunnarsson, and A.I. Liechtenstein, Phys. Rev. B 46, 1773 (1992).

15 I.I. Mazin, A.I. Liechtenstein, O. Gunnarsson, O.K. Andersen, V.P. Antropov, and S.E. Burkov, Phys. Rev. Lett. 26, 4142 (1993).

${ }^{16}$ M.S. Deshpande, E.J. Mele, M.J. Rice, and H-Y. Choi, Phys. Rev. B 50, 6993 (1994). In this paper the optical conductivity was also calculated for intermediate frequencies using a diagram which gives a small contribution for a disordered solid, while the Drude peak was not studied.

${ }^{17}$ Lannoo, M., G.A. Baraff, M. Schluter, and D. Tomanek, 1991, Phys. Rev. B 44, 12106.

${ }^{18}$ O. Gunnarsson, Phys. Rev. B 51, 3493 (1995).

19 G. Czycholl and H.J. Leder, Z. Phys. B 44, 59 (1981).

${ }^{20} \mathrm{G}$. Grimvall, The electron-phonon interaction in metals, North-Holland, (1981), p. 212.

21 A.I. Liechtenstein, O. Gunnarsson, M. Knupfer, J. Fink, and J.F. Armbruster, J. Phys.: Cond. Matter 8, 4001 (1996).

22 O. Gunnarsson, H. Handschuh, P.S. Bechthold, B. Kessler, G. Ganteför, and W. Eberhardt, Phys. Rev. Lett. 74, 1875 (1995).

${ }^{23}$ K. Prassides, C. Christides, M.J. Rosseinsky, J. Tomkinson, D.W. Murphy, and R.C. Haddon, Europhys. Lett. 19, 629 (1992).

${ }^{24}$ Winter, J. and H. Kuzmany, 1996, Phys. Rev. B 53, 655.

${ }^{25}$ R.L. Martin and J.P. Ritchie, Phys. Rev. 48, 4845 (1993).

${ }^{26}$ D.P. Joubert, J. Phys.: Condens. Matter 5, 8047 (1993).

${ }^{27}$ F. Aryasetiawan, O. Gunnarsson, E. Koch, and R.M. Martin, Phys. Rev. B (submitted).

${ }^{28}$ R.D. Cowan, The theory of atomic structure and spectra, Univ. of California Press, Berkley, 1981.

${ }^{29}$ A.I. Liechtenstein, I.I. Mazin, O. Gunnarsson, O.K. Andersen, S.E. Burkov, and V.P. Antropov, in Electronic Properties of Fullerenes, Proceedings of the International Winterschool on Electronic Properties of Novel Materials, Kirchberg, 1993, Editors H. Kuzmany, J. Fink, M. Mehring, and S. Roth, Springer (Berlin, 1993), p. 211.

${ }^{30}$ S. Teslic, T. Egami, and J.E. Fischer, Phys. Rev. B 51, 5973 (1995).

${ }^{31}$ E. Sohmen, J. Fink, and W. Krätschmer, Europhys. Lett. 17, 51 (1992).

32 O. Gunnarsson, V. Eyert, M. Knupfer, J. Fink, J.F. Armbruster, J. Phys.: Condens. Matter 8, 2557 (1996).

${ }^{33}$ O. Gunnarsson, (to be publ.)

${ }^{34}$ L. Hedin, Phys. Rev. 139, A796 (1965).

35 A.P. Ramirez, M.J. Rosseinsky, D.W. Murphy, and R.C. Haddon, Phys. Rev. Lett. 69, 1687 (1992).

${ }^{36}$ G.J. Burkhart, and C. Meingast, Phys. Rev. B 54, R6865 (1996). 